\title{
SELEÇÃO DE PERFIL DE PALESTRANTE ESPECIALISTA PARA OFICINA DE FORMAÇÃO DOCENTE ${ }^{1}$
}

\author{
Sarah Francisca de Souza Borges ${ }^{\mathrm{a} *}$, Moacyr Machado Cardoso Junior ${ }^{\mathrm{a}}$, Mischel Carmen \\ Neyra Belderrain ${ }^{\mathrm{a}}$ \\ anstituto Tecnológico de Aeronáutica, Ciências e Tecnologias Espaciais, \\ São José dos Campos-SP, Brasil
}

Recebido 15/06/2020, aceito 07/11/2021

\begin{abstract}
RESUMO
As contratações em geral precisam ser conduzidas com muita cautela e probidade, dentro de normas e leis, refletindo em responsabilidades para ambas as partes. O objetivo deste artigo foi a seleção de perfil de palestrante especialista para uma oficina de formação docente. Com base na revisão bibliográfica foram selecionados os métodos: 1) VFB (Value Focused Brainstorming) para a estruturação do problema, bem como identificar e alinhar objetivos, critérios e alternativas. 2) FITradeoff (Flexible and Interactive Tradeoff) para comparação de alternativas no processo de elicitação e exigindo menos informações do decisor. Quanto as fases da decisão, o método VFB foi utilizado para "Estruturação da situação problemática" e "Estruturação do modelo de avaliação MCDA"; e o método FITradeoff para "Modelagem de preferências e avaliação de alternativas de decisão". Como resultado, a integração de métodos facilitou o processo da definição de objetivos até seleção da alternativa ótima que representa o melhor perfil do palestrante.
\end{abstract}

Palavras-chave: Multicritério, VFB, FITradeoff, Decisão.

\begin{abstract}
Hiring in general needs to be conducted with great caution and probity, within rules and laws, reflecting responsibilities for both parties. The purpose of this article was to select the profile of an expert speaker for a teacher training workshop. Based on the literature review, the following methods were selected: 1) VFB (Value Focused Brainstorming) for structuring the problem, as well as identifying and aligning objectives, criteria, and alternatives. 2) FITradeoff (Flexible and Interactive Tradeoff) for comparing alternatives in the elicitation process and requiring less information from the decision-maker. As for the decision phases, the VFB method was used for "Structuring the problematic situation" and "Structuring the MCDA evaluation model"; and the FITradeoff method for "Modeling preferences and evaluating decision alternatives". As a result, the integration of methods facilitated the process of setting goals until selecting the optimal alternative that represents the best profile of the speaker.
\end{abstract}

Keywords: Multicriteria, VFB, FITradeoff, Decision.

*Sarah Francisca de Souza Borges. E-mail: $\underline{\text { sarah@ita.br. }}$

DOI: 10.4322/PODes.2022.001

${ }^{1}$ Todos os autores assumem a responsabilidade pelo conteúdo do artigo. 


\section{Introdução}

No contexto do mercado competitivo, em um ambiente acirrado e complexo, as empresas têm centrado atenções em suas atividades principais, onde detêm sua expertise. Assim, os processos de suporte a gestão empresarial passam a ser contratados por especialidade com empresas prestadoras de serviços ou profissionais especialistas. São as práticas da terceirização dos serviços não afins. Embora estes sejam chamados de serviços secundários, podem envolver altos riscos para a empresa contratante, no caso de saírem do controle e incorrer a algum desvio daquilo do que foi contratado poderá trazer relevantes e irrecuperáveis prejuízos à empresa. Logo, os gestores devem munir-se de técnicas e ferramentas para seleção do profissional e elaboração de um contrato mais completo possível, posteriormente acompanhando a evolução física e financeira da prestação dos serviços durante toda a sua vigência, objetivando minimizar os riscos (Madeira e Palmeira, 2011).

Nesse sentido, a contratação precisa ocorrer dentro de normas e leis, e na seleção de qualquer prestador de serviços precisa haver cautela e probidade, pois gera impacto na organização contratante. $\mathrm{O}$ desempenho de uma empresa ou profissional prestador de serviços sobre uma organizaoção refletirá em resultados e responsabilidades para ambas as partes, aumentando a relevância de métodos multicritérios de apoio à decisão na especificação de um perfil demandante. Segundo Bana e Costa e Vincke (1990), a vantagem da utilização de métodos multicritérios, em geral, ocorre por que não há decisões que sejam simultaneamente ótimas sob todos os pontos de análise, fazendo-se necessário a seleção da melhor opção possível.

Além disso, as exigências de justificativa do processo decisório se tornam cada vez mais complexas em virtude da quantidade e natureza dos critérios considerados na avaliação. Estes critérios quando não são bem definidos, podem inviabilizar todo um processo de licitação, comprometendo recursos e qualidade do serviço público ou privado. Assim, o processo decisório precisa ser eficiente e eficaz, e a decisão final precisa refletir ao atendimento do interesse público.

O objetivo deste artigo foi a seleção de perfil de palestrante especialista para uma oficina de formação docente em uma Universidade. Tratava-se de um processo licitatório, abrangendo menor preço e melhor técnica, ou seja, a montagem da especificação demandava previamente a clara definição dos critérios e descrição da alternativa ótima do perfil, pois, uma vez definidos, estes seriam publicados oficialmente e alterações posteriores poderiam ser prejudiciais ou inaceitáveis.

Com o intuito de estruturação de objetivos, identificação de critérios e especificação da melhor alternativa, este trabalho contribui no estudo da integração dos métodos VFB (Value Focused Brainstorming) (proposto por Keeney em 2012) e FITradeoff (Flexible and Interactive Tradeoff) (proposto por Almeida et al. em 2016). Com o método VFB é possível identificar o real problema de decisão, objetivos e gerar as melhores alternativas; com o método FITradeoff é possível verificar qual alternativa melhor atende aos critérios sem requerer muitas informações do decisor. Além disso, com a comparação das alternativas segundo a perspectiva do decisor é possível entender o processo até a solução mais adequada e verificar o quanto os métodos de apoio a decisão justificam a estratégia tomada.

Após esta introdução, este artigo está estruturado na sequência: A segunda parte, o referencial teórico, com a estrutura do processo decisório adotado e pesquisa bibliográfica de trabalhos que utilizaram os mesmos métodos, bem como a explicação do conceito e como aplicar cada um. A terceira parte são os resultados e discussões, abordando a necessidade de especificação de um perfil de palestrante especialista para uma palestra com o tema Conception, Design, Implementation and Operation-CDIO. A quarta e última parte é a conclusão, destacando os pontos fortes e fracos de cada método, bem como a viabilidade da proposta de integração desses. 


\section{Referencial Teórico}

Poderiam ser utilizados diferentes métodos para a problemática apresentada (áreas de pesquisa como: gestão de projetos, estruturação de problemas, análise de decisão, etc.), contudo, foi considerado quais métodos nos próprios passos auxiliavam no processo decisório de definição de critérios e seleção da alternativa ótima (contemplando a análise do problema, definição de objetivos e critérios, identificação e seleção de alternativa). Buscou-se também o menor número de métodos combinados.

Assim, focou-se no estudo dos Métodos Multicritério de Apoio à Decisão (AMD) ou Multi-Criteria Decision Aiding (MCDA), sugeridos por Franco e Montibeller (2011) para uma visão geral e estruturação do problema em estudo, ao considerar múltiplos objetivos e a complexidade.

Para Holanda (2017), diariamente as pessoas são expostas a problemas e acabam tomando decisões intuitivamente. No entanto, quando estas decisões se tornam mais complexas, envolvendo consequências com alto risco, faz-se necessário utilizar um modelo de estruturação de problemas para auxiliar no processo de tomada de decisão. A estruturação de um problema é uma das etapas do processo de decisão, que tem como objetivo auxiliar na estruturação da situação problemática, para as quais se buscam propostas alternativas.

Assim, para atingir o objetivo são utilizados critérios, em que o estudo do desempenho leva a avaliação das alternativas. No processo de decisão, o facilitador/analista (podendo ser um consultor) é muito útil no suporte metodológico para o Tomador de decisão em todos os estágios, e trabalha na estruturação do problema e construção do modelo (Almeida et al., 2015).

O processo de apoio multicritério à decisão considera três fases: Estruturação da situação problemática, Estruturação do modelo multicritério de avaliação MCDA, e Modelagem de preferências e avaliação de alternativas de decisão (Figura 1) (Franco e Montibeller, 2011).

Figura 1: Estrutura do processo de apoio multicritério à decisão MCDA.

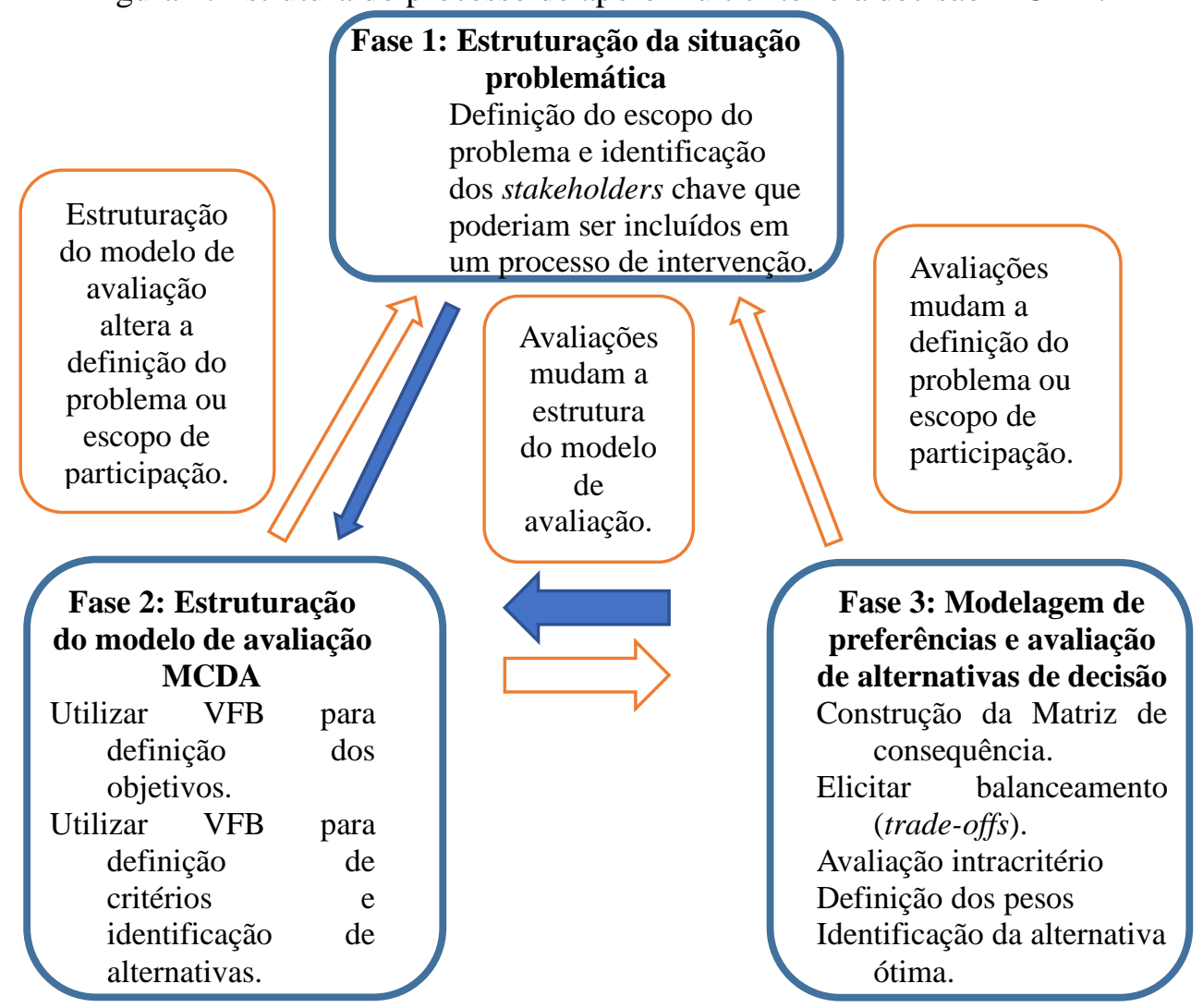

Fonte: Adaptado de Franco e Montibeller (2011). 
Neste artigo buscou-se qual(is) método(s) atenderia(m) as três fases. Dentro do objetivo da pesquisa, foram selecionados: o método VFB para "Estruturação da situação problemática" e "Estruturação do modelo de avaliação MCDA"; e o método FITradeoff para "Modelagem de preferências e avaliação de alternativas de decisão".

Considerando os benefícios de cada método neste campo de estudo, destacaram-se os métodos VFB e FITradeoff.

O VFB tem como vantagem incorporar a técnica Brainstorm na inicial sugestão de alternativas individualmente pelos participantes (antes da decisão em grupo) e agrega valor ao permitir explorar a criatividade no processo decisório (Keeney, 2012). Sendo também mais recente, o VFT foi proposto por Keeney em 1992 (Keeney, 1992) e o VFB pelo mesmo autor foi proposto em 2012 (Keeney, 2012).

Quanto ao FITradeoff, segundo Frej (2019) apresenta significativa vantagem em comparação a outras abordagens baseadas em tradeoffs, com um procedimento de elicitação estruturado para conseguir as informações do decisor, ao contrário da maioria dos métodos de informação parcial presentes na literatura que o fazem de maneira arbitrária. Além disso, com o FITradeoff, diferentemente do procedimento de tradeoff tradicional, o decisor não precisa estabelecer pontos exatos que tornam duas consequências indiferentes para ele, sendo assim, é cognitivamente de mais fácil entendimento e leva a uma menor taxa de inconsistências no processo de elicitação. Também ressalta-se que o método FITradeoff foi vencedor do prêmio de melhor produto tecnológico no EPPGEP 2017, da ANPEPRO, e ganhou o prêmio EURO Working Group on Decision Support Systems 2017 Award, na ICDSST 2017.

\subsection{Bibliometria dos Métodos VFT ou VFB e FITradeoff}

A princípio, foi realizado uma pesquisa bibliométrica em maio de 2020, com os termos "VFB" e "FITradeoff" nas bases de dados Google Escolar, SCOPUS e Web of Science (no resumo, título e palavras chave) e não foi encontrado resultado. Nas duas últimas bases, com o termo "Value Focused Brainstorming", foram encontrados apenas três artigos. Sendo eles:

1. O artigo Value-Focused Brainstorming da revista Decision Analysis, trata da necessidade de melhorar a qualidade e a capacidade de inovação na identificação de alternativas, o VFB incorpora duas características do pensamento focado no valor nos procedimentos tradicionais de brainstorming. Uma aplicação de política pública, feita para tratar de recomendações após o desastre do World Trade Center, ilustra a abordagem criando alternativas para melhorar a evacuação de emergência de indivíduos de grandes edifícios (Keeney, 2012).

2. O artigo "A systemic framework based on Soft OR approaches to support teamwork strategy: An aviation manufacturer Brazilian company case", publicado no Journal of the Operational Research Society. Apresenta uma combinação de abordagens de Pesquisa Operacional (OR) para o caso real de redesenhar a estratégia de uma equipe de gerenciamento do departamento de fabricação de aeronaves no Brasil. O framework proposto utiliza Strategic Options Development and Analysis (SODA) para entender e estruturar a situação; Value-Focused Thinking (VFT) para definir os objetivos meios e fins; Value-Focused Brainstorming (VFB) para destacar as soluções potenciais (Abuabara et al., 2018).

3. Artigo de Ralph L. Keeney, que apresenta Value-Focused Brainstorming, na revista Decision Analysis.

Quanto aos termos "value-focused thinking" e "Fitradeoff", na base Web of Science foram encontrados 3 trabalhos e na base Scopus foram 5 trabalhos, estes estão enquadrados nas áreas de análise de decisão e engenharia. Destacam-se os artigos:

- Integrating value-focused thinking and FITradeoff to support information technology outsourcing decisions, com o objetivo de propor uma estrutura combinando a metodologia de pensamento focado em valor (VFT) e o método FITradeoff para apoiar decisões relacionadas a terceirização de tecnologia da informação (information technology outsourcing, ITO) (Poleto et al., 2020). 
- The integration of VFT and FITradeoff multicriteria method for the selection of WCM projects, com o objetivo de propor o uso do método de estruturação de problemas do pensamento focado no valor (VFT) com o método FITradeoff para fornecer maior coerência e eficiência ao longo do processo de seleção de projetos de manufatura de classe mundial (world class manufacturing, WCM) (Silva et al., 2019).

Com o termo "FITradeoff" foram encontrados 22 trabalhos no Web of Science e 38 no Scopus, principalmente na área de Engenharia, Ciência da decisão, Matemática e Ciência Computacional. Destes, quanto a integração de métodos, foi encontrado apenas entre VFT e o FITradeoff, sendo os artigos citados anteriormente.

Assim, por meio da pesquisa bibliográfica foi possível identificar os autores e os trabalhos sobre os métodos, possibilitando o estudo de aplicações e descrição dos passos.

A integração dos métodos VFT e FITradeoff já ocorreu, mas neste trabalho busca-se avançar no estudo de integração dos métodos VFB e FITradeoff, não encontrado semelhante na literatura.

\subsection{Fases 1 e 2 do Processo de Apoio Multicritério à Decisão MCDA}

Para a Fase 1 (Estruturação da situação problemática) e a Fase 2 (Estruturação do modelo de avaliação MCDA) foram utilizados os Métodos de Estruturação de Problemas (PSM Problem Structuring Methods), pois permitem que várias perspectivas sejam trabalhadas em conjunto, sendo acessível a usuários que não tenham um treinamento especializado, uma vez que sua base matemática é simples e de fácil entendimento. Assim, o desenvolvimento da estruturação ocorre em um processo participativo entre os integrantes envolvidos (Mingers e Rosenhead, 2004).

Os PSMs mais conhecidos e utilizados são: SSM (Soft Systems Methodology), SODA (Strategic Options Development and Analysis), VFT (Value-Focused Thinking) e SCA (Strategic Choice Approach) (Andrade e Morais, 2016.

Segundo Andrade e Morais (2016) e Keeney (2012), quando há uma decisão bastante complexa, com objetivos que não estão claros e quando as alternativas não são conhecidas pelo grupo, uma abordagem VFT pode conduzir a uma melhor solução no momento de tomada de decisão.

De acordo com Keeney (1996) os enfoques convencionais para a tomada de decisão tratam da geração e avaliação de alternativas, abordagem denominada pelo autor como Alternative Focused Thinking (AFT). Ou seja, os decisores concentram-se primeiro nas alternativas e somente depois identificam os objetivos ou critérios para avaliá-los.

O AFT representa uma abordagem limitada para a tomada de decisões, pois as alternativas são relevantes apenas por integrarem os meios no processo de atingir algo mais importante: os valores. Os valores constituem princípios para se avaliar o quanto se deseja de uma possível alternativa ou consequência, e definem o que é mais relevante dentro de uma decisão (Keeney, 1994). Neste sentido, os valores (e não as alternativas) deveriam ser a força direcionadora de todo processo de tomada de decisão. Este conceito focado em valores é a base do método VFT proposto por Keeney $(1992,1994,1996)$.

Neste mesmo sentido, em 2012, Keeney propos o VFB (Value Focused Brainstorming), integrando o VFT à técnica brainstorming. Segundo o autor, o brainstorming é uma técnica utilizada na busca de alternativas para decisões complexas. Contudo, é comum en contrar pouco eficácia. No sentido de melhorar a qualidade e inovação dos elementos de decisão, o VFB incorpora características do brainstorming tradicional, agregando novos elementos que permitem a combinar a criatividade e assertividade na geração de ideias (Keeney, 2012).

Além disso, na integração do brainstorming com o pensamento focado em valor tem-se uma maior identificação explicita dos aspectos valorizados das alternativas e os participantes individualmente criam alternativas antes de qualquer ancoragem nas discussões em grupo, o que aumenta o alcance da articulação dos pensamentos de cada indivíduo (Keeney, 2012).

Assim, na composição deste artigo foi selecionado o VFB, sendo mais recente e buscando explorar a identificação de mais alternativas. A Figura 2 apresenta os passos do VFB. 
Figura 2: Passos do VFB.

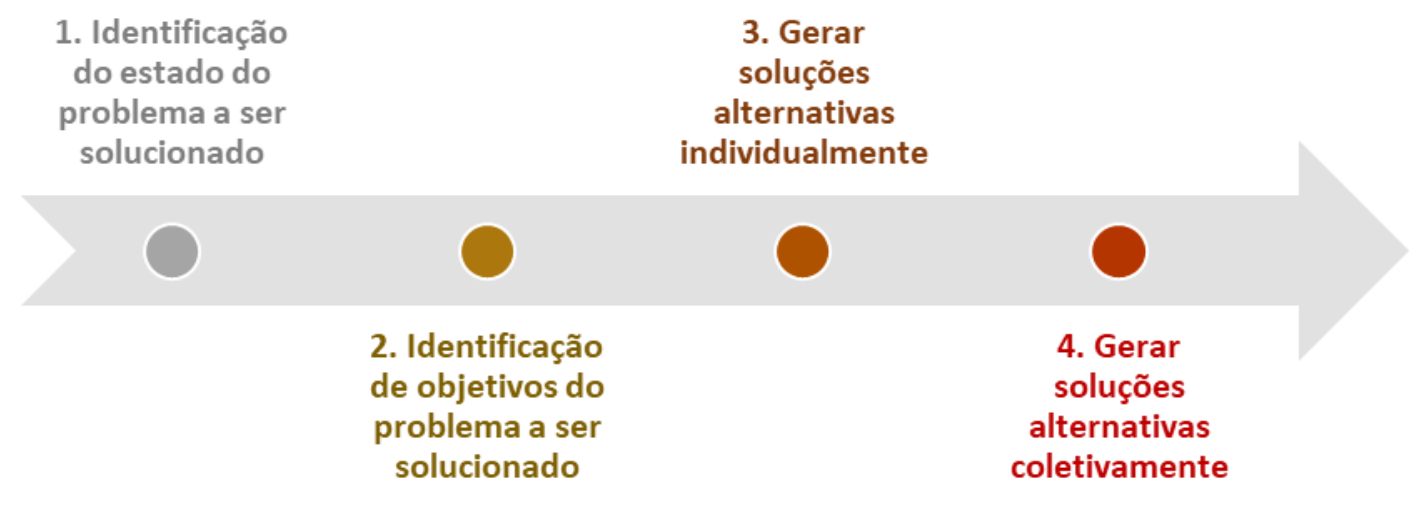

Fonte: Keeney (2012).

Muitos pesquisadores reconhecem que um dos requisitos básicos para a tomada de boas decisões é uma lista completa de objetivos, visando entender o que precisa ser melhorado (Keeney, 1992; Keeney e Raiffa, 1993). Por outro lado, a identificação e estruturação dos objetivos em um processo de tomada de decisão pode representar um grande desafio, em que frequentemente objetivos fins são confundidos com objetivos meios, os objetivos são confundidos com restrições ou alternativas, e as relações entre diferentes objetivos não são especificadas (Gonçalves, 2010; Keeney, 1996).

Segundo Keeney (1992): Objetivos Estratégicos são objetivos essenciais que direcionam as decisões organizacionais e são utilizados para se tomar decisões no nível estratégico de uma organização. Objetivos Fundamentais representam os fins que os decisores almejam em um contexto de decisão. Objetivos Meios representam as maneiras para se alcançar outros objetivos.

Ressalta-se que as fases 1 e 2 do MCDA foram explicadas em conjunto por serem abrangidas no mesmo método (VFB), contudo, apresentam resultados diferentes, conforme Figura 1.

\subsection{Fase 3 do Processo de Apoio Multicritério à Decisão MCDA}

Segundo Almeida (2013), na definição de peso dos critérios, na decisão multicritério, principalmente em modelos aditivos, um problema é a dificuldade do Decisor em entender o significado do peso ou não possuir conhecimento e informações suficientes para defini-los. Devido a dificuldades e desafios importantes ao definir os pesos, o procedimento de compensação do método tradeoff revelou-se base de raciocínio com forte fundamentação axiomática.

Para a Fase 3 (Modelagem de preferências e avaliação de alternativas de decisão) foi selecionado o método FITradeoff (Flexible and Interactive Tradeoff), pois trata-se de um método que permite a comparação de alternativas e melhora o processo de licitação (Almeida et al., 2016a).

O método FITradeoff foi desenvolvido para elicitar pesos de critérios dentro do escopo da Teoria de Valor Multicritério, baseada em tradeoffs. A principal característica desse novo método, comparado ao procedimento de compensação tradicional, é que trabalha com informações parciais sobre as preferências do Decisor e requer menos esforço cognitivo durante o processo de elicitação (Frej et al., 2017). Assim, trata-se de uma ferramenta útil para avaliação compensatória das alternativas com a vantagem de requerer menos informações por parte do decisor (Camilo et al., 2020; Holanda, 2017).

Além disso, este método funciona com base no conceito de otimização para escolha da melhor alternativa em um conjunto determinado de ações possíveis, baseado em julgamentos de 
tradeoff fornecidos pelo Decisor. A elicitação é realizada de forma interativa por meio de um Sistema de Suporte à Decisão, que fornece a visualização gráfica para auxiliar na análise de resultados parciais (Frej et al., 2017).

A modelagem de preferência do Decisor e a junção destas no modelo de decisão são considerados desafios nos problemas de decisores (Almeida, 2013; Figueira et al., 2016; Macedo et al., 2018). Com o método FITradeoff o número de alternativas não é uma questão relevante, pois considera uma racionalidade compensatória e logo no início busca otimizar o número de alternativas baseado em simulações (Almeida, 2013; Macedo et al., 2018). Este método foi desenvolvido para obter constantes de escala $\left(k_{i}\right)$ de critérios dentro do escopo da Teoria de Valor Multiatributo (Multiple-Attribute Value Theory, MAVT), em que as alternativas $a_{j}$ são pontuadas diretamente de acordo com a função valor na equação (1) (Frej et al., 2017):

$$
v\left(a_{j}\right)=\sum_{i=1}^{n} k_{i} v_{i}\left(a_{j}\right)
$$

Sendo que $v(a)$ é o valor da alternativa $a$ para o decisor, $k_{i}$ é o peso do critério $i$ e $v_{i}\left(a_{j}\right)$ é o valor da consequência $a$ para o critério $i$. A depender da estrutura de preferências do decisor, $v_{i}\left(a_{j}\right)$ pode assumir uma função linear ou não linear. Considerando um problema de decisão multicritério com $m$ alternativas e $n$ critérios e um decisor, o método FITradeoff segue os passos descritos a seguir (Almeida et al., 2016b).

O primeiro passo é a classificação dos pesos dos critérios, equação (2). Esta etapa é conduzida da mesma maneira que o procedimento tradicional de tradeoff, considerando $k_{i}$ como o critério mais preferível e $k_{n}$ como o critério menos preferível, a seguinte relação é obtida após essa etapa (Frej et al., 2017):

$$
k_{1}>k_{2}>k_{3}>k_{4}>\cdots>k_{n}
$$

O procedimento de tradeoff tradicional exige que o decisor especifique o exato ponto para o qual o decisor é indiferente entre duas alternativas $x_{i}^{I}$, para formar equações do tipo (3) e assim resolver um sistema de equações encontrando os valores constantes de escala. Contudo, esta informação de ponto de indiferença é cognitivamente difícil de ser fornecida, o decisor pode até não saber fornecê-la de maneira consistente. Considerando estas questões, o FITradeoff trabalha apenas com questões em que o decisor deve fornecer informações sobre preferência estrita sobre as alternativas, de forma que inequações do tipo (4) e (5) sejam obtidas. Destaca-se que estas inequações não permitem calcular valores exatos para os pesos, mas elas indicam os limites superior $x_{i}^{U} \mathrm{e}$ inferior $x_{i}^{L}$ entre os valores, de maneira que um espaço de pesos de possíveis vetores admissíveis de pesos $k$ é obtido (Frej, 2019).

$k_{i} v_{i}\left(x_{i}^{n}\right)=k_{i+1}$

$k_{i} v_{i}\left(x_{i}^{U}\right)>k_{i+1}$

$k_{i} v_{i}\left(x_{i}^{L}\right)<k_{i+1}$

Além disso, no software FITradeoff são estabelecidas restrições às declarações de preferência estritas do decisor ao comparar as alternativas. $\mathrm{O}$ valor $\delta$ pode ser considerado um valor muito pequeno que estará na inequação apenas para efeitos computacionais da programação linear, visa que (6) e (7) sejam inequações equivalentes a (3) e (4) respectivamente (Frej, 2019).

$k_{i} v_{i}\left(x_{i}^{\prime}\right) \geq k_{i+1}-\delta, i=1 a n-1$

$k_{i} v_{i}\left(x_{i}^{\prime \prime}\right) \geq k_{i+1}+\delta, i=1 a n-1$ 
A fim de verificar a alternativa ótima $a_{j}$ neste ponto, o valor é maximizado como um modelo de Problema de Programação Linear, com a função objetivo definida na equação (8), onde as variáveis de decisão são as constantes de escala $a_{j}$ sujeitas às restrições das equações (6) e (7) e considerando pesos normalizados (9) e não-negativos (10) (Frej et al., 2017).

$$
\begin{aligned}
& \max \sum_{i=1}^{n} k_{i} v_{i}\left(x_{i j}\right) . \\
& \sum_{i=1}^{n} k_{i}=1 \\
& k_{i} \geq 0, i=1 \ldots n
\end{aligned}
$$

No segundo estágio da aplicação, o analista conduz a modelagem de preferência com o Decisor usando o FITradeoff, um procedimento que lida com alguma inconsistência e complexidade observada no tradicional procedimento de tradeoff (Almeida et al., 2016a; Macedo et al., 2018). O espaço de peso preliminar (2) é obtido e a otimização potencial de cada alternativa será verificada por problemas de programação linear a fim de identificar quais alternativas são potencialmente ótimas para o problema. Uma alternativa é potencialmente ótima se seu valor em (1) for maior ou igual aos valores de todas as outras alternativas para pelo menos um vetor de peso dentro do espaço de pesos $z$; isto é, uma alternativa $a_{j}$ é potencialmente ótima se a seguinte desigualdade for satisfeita (11) (Frej et al., 2017):

$$
\sum_{i=1}^{n} k_{i} v_{i}\left(x_{i j}\right) \geq \sum_{i=1}^{n} k_{i} v_{i}\left(x_{i z}\right) \forall_{z}=1, \ldots, m, z \neq j
$$

Sendo que $v_{i}\left(x_{i z}\right)$ é o valor do desempenho $x$ para o critério $i$ em sua função intracriterio dentro do intervalo entre 0 e 1 , referente ao desempenho dentre as alternativas (Almeida et al., 2016b)

A função objetivo (8) visa maximizar o valor global (1) da alternativa $a_{j}$, e as variáveis de decisão são as constantes de escala $k_{i}$. A primeira restrição (2) trata da ordenação de constantes de escala, estabelecida pelo decisor por avaliação holística ou por comparação par a par. As restrições (6) e (7) vem das declarações de preferência estrita estabelecidas pelo decisor ao comparar as alternativas hipotéticas. As inequações em (11) garantem a potencial otimicidade, ou seja, a restrição só será satisfeita se houver ao menos um vetor de pesos $k$ para o qual o valor global de $a_{j}$ é maior ou igual ao valor global de todas as outras alternativas. A restrição (9) garante que os pesos são normalizados e é assegurada a não-negatividade destes valores (10). Assim, pode-se dizer que a alternativa $a_{j}$ é potencialmente ótima se e somente se o modelo de programação linear tiver solução viável, ou seja, se dentro do espaço de pesos delimitado pelas restrições (2,6-7,9-10), houver ao menos um vetor de pesos que satisfaça as restrições em (11). Caso a PPL para $a_{j}$ não admita solução viável, $a_{j}$ é eliminada do processo. Caso contrário, $a_{j}$ está dentro da solução viável e continuará como candidata a alternativa ótima do problema (Frej, 2019).

Para fechar o referencial teórico, o presente estudo seguirá as fases da Figura 1, primeiramente será realizado a estruturação da situação problemática (definindo o problema e identificado os Stakeholders), Estruturação do modelo multicritério (definindo objetivos, critérios e alternativas) e Avaliação de Alternativas (elicitação para busca da alternativa ótima por meio do FITradeoff). Este processo de apoio multicritério à decisão descrito será aplicado ao problema de contratação de palestrante especialista para promover oficinas de formação docente em uma universidade.

\section{Resultados e Discussões}

Este estudo trata do momento de contratação de palestrante especialista por meio de recurso público, envolvendo: 1) Ministério da Ciência, Tecnologia, Inovações e Comunicações (administração direta do governo federal do Brasil que transmite recursos a projetos de pesquisa 
e investiria nesta iniciativa); 2) Instituição de ensino superior (demandante do serviço); 3) Fundação de apoio (órgão responsável por realizar a contratação). MCDA.

A seguir serão desenvolvidas as três Fases do Processo de apoio multicritério à decisão

\subsection{Fase 1: Estruturação da Situação Problemática}

Para entender o problema, foi realizada uma análise de Stakeholders e definido três (nessa ordem): Analista da área de Compras e Projetos (que acompanharia o processo de contratação); Professor da Instituição na área de Gestão de Riscos (provável participante da oficina) e Coordenador de Projeto (principal decisor na especificação da contratação).

A princípio, foram realizadas entrevistas individuais com algumas questões que poderiam nortear o problema como apresentado na Tabela 1. No decorrer do trabalho, o contato foi maior com o Coordenador de Projeto, e ao final foi apresentado o resultado a todos.

Nesse sentido, a declaração do problema é o passo inicial do VFB, pois quando bem aplicado inclinará o grupo a ter alternativas assertivas e coerentes com a solução esperada. Assim, nesta fase do VFB é imprescindível compreender com clareza o problema a ser resolvido (Souza Junior et al., 2015).

O próximo passo do VFB visa identificar a situação alvo, o que de fato objetiva-se e onde se pretende chegar com as alternativas geradas. Nesta fase busca-se resolver o problema declarado, expondo os objetivos de modo a ter todos os indivíduos devidamente alinhados com o que se espera (Souza Junior et al., 2015).

Tabela 1: Questões base para entrevista.

\section{Questionário}

1. Qual a necessidade/problema apresentado?

2. Quais as possíveis alternativas? Qual seria a perfeita, a pior e a razoável?

3. Quais são os seus valores absolutamente fundamentais?

4. Siga as relações meio-fim: Por que aqueles objetivos são importantes? Como faria para alcançá-los?

5. Como você mede o atendimento de um objetivo? Porque o objetivo A é mais importante do que o objetivo B?

$$
\text { Fonte: Adaptado de Keeney (1996). }
$$

Tais perguntas apoiaram no melhor entendimento e direcionamento à estruturação do problema. Pois, serviriam de base para identificar objetivos estratégicos, objetivos fundamentais e objetivos meios, os quais puderam ser hierarquizados.

Os valores vistos como pilares para nortear a estruturação do problema e para desenvolver o processo de análise foram: Transparência, comprometimento e profissionalismo.

\subsection{Fase 2: Estruturação do Modelo de Avaliação MCDA}

Com base no questionário, foi possível verificar que a necessidade principal era a contratação de um profissional como palestrante de uma oficina de formação docente na Instituição. Uma preocupação era especificar os critérios, que precisariam ser publicados em edital, bem como definir o perfil mais próximo demandado. Quanto a este perfil foi possível determinar requisitos, tidos como alternativas.

Outros pontos apontados na entrevista foram: 1) Interesse de que o palestrante fosse externo à Instituição para obter uma nova perspectiva. 2) Possíveis temas para oficina docente (demandados pelos docentes e órgãos superiores). 3) Necessidade de avaliar as restrições para vinda do palestrante (principalmente quanto ao valor da passagem e disponibilidade de data). 4) Necessidade de verificar o nível de apoio administrativo necessário para trazer o palestrante.

Ao final desta análise, o Coordenador do Projeto (com base nas sugestões das entrevistas individuais com os demais Stakeholders) definiu o tema de Novas Metodologias de Ensino, 
especificamente Conception, Design, Implementation and Operation-CDIO. Foi estabelecido apenas um dia de oficina (sendo que, quanto maior a disponibilidade do Palestrante especialista, melhor).

A seleção de perfil de palestrante especialista foi definida como objetivo estratégico para continuar a análise.

Para diferenciar os objetivos fundamentais e objetivos meios utilizou-se a técnica WITI Why is this important? (“por que isso é importante?"). Segundo Keeney (1996) é necessário questionar os stakeholders sobre a importância daquele objetivo.

Conforme proposto por Keeney (1992), nesta etapa também foi verificado se os objetivos atendiam propriedades como: essencial, controlável, mensurável, não-redundante, conciso e entendível.

Assim, após a identificação dos objetivos fundamentais e meios, foi elaborado uma hierarquia entre os mesmos com propósito de orientar o decisor. A Tabela 2 apresenta os objetivos fundamentais e meios hierarquizados.

Tabela 2: Hierarquização de objetivos.

\section{Objetivos fundamentais e meios}

1 Profissional palestrante com menor custo.

1.1 Buscar menor custo de passagem;

1.2 Buscar menor custo de diária;

1.3 Buscar menor custo de valor-hora.

1.3.1 Menor valor do transporte;

1.3.1 Menor valor da alimentação;

1.3.1 Menor valor da hospedagem.

2 Profissional com maior carga horária disponível.

2.1 Pesquisar profissional com maior carga horária disponível.

3 Profissional com perspectiva de gerar maior impacto.

3.1 Pesquisar nível de expectativa esperada com base no histórico.

4 Profissional com menor risco.

4.1 Pesquisar profissional com maior confiabilidade de aceite do convite;

4.2 Pesquisar profissional com maior confiabilidade de comparecimento.

4 Profissional com maior número de publicações.

4.1 Pesquisar profissional com mais publicações em congressos, nacionais e internacionais, revistas, dissertações, teses, entre outros.

5 Profissional com maior experiência docência.

5.1 Pesquisar profissional com maior carga horária e anos em atividades docentes.

6 Profissional com maior número de projetos no tema.

6.1 Pesquisar o profissional com mais projetos de pesquisa no tema.

Fonte: Elaborado pelos autores.

A Figura 3 apresenta a Rede de objetivos Meio-Fim construída a partir da Hierarquização de Objetivos. Esta representa a consolidação de informações das entrevistas, foi enviada individualmente aos stakeholders e aprovada sem divergências (pois as ideias de todos foi acrescentada e trabalhou-se mais na terminologia). 
Figura 3: Rede de objetivos meio-fim.

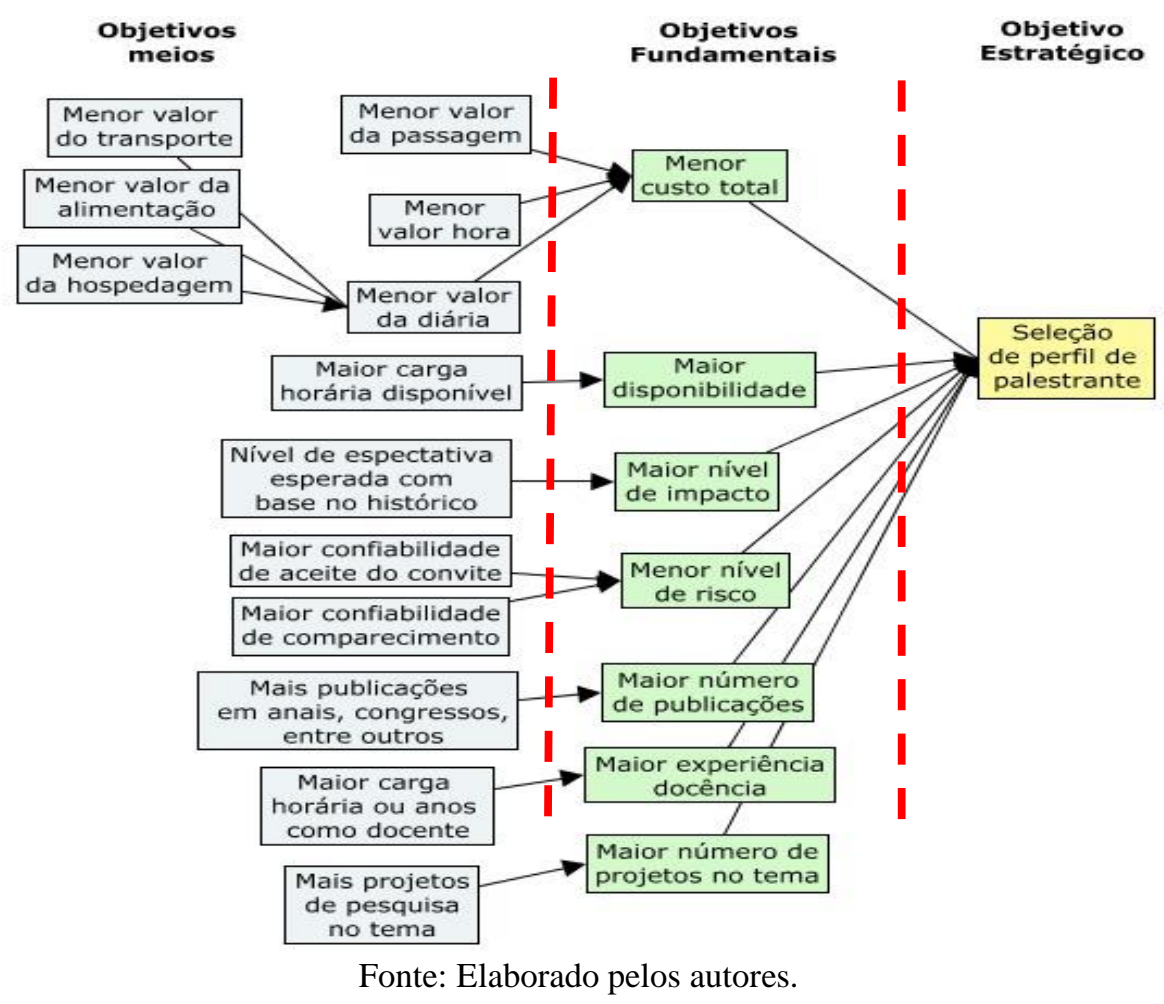

Com base no questionário individual foi possível identificar as alternativas possíveis. Estas depois foram refinadas e validadas em uma entrevista em grupo.

Ao final, foram obtidos seis perfis de profissionais: Palestrante A - nacional (professor de universidade em Curitiba); Palestrante B - nacional (professor de universidade em Brasília); Palestrante C - internacional (professor de universidade em Singapura); Palestrante D internacional (professor do MIT); Palestrante colaborador - nacional (professor de universidade em São Paulo); Palestrante colaborador - internacional (professor de universidade nos Estados Unidos).

Assim, bastava-se testar os critérios em relação as diferentes alternativas de perfis, ou seja, poderiam ser profissionais de diferentes instituições e localidades (o intuito não era induzir a contratação do profissional, mas o perfil que melhor atendesse a demanda).

\subsection{Fase 3: Modelagem de Preferências e Avaliação de Alternativas de Decisão}

Para esta aplicação, a identificação de objetivos auxiliou na construção da Árvore de Valor, com definição do objetivo principal, critérios e alternativas (conforme Figura 4). 
Figura 4: Árvore de valor.

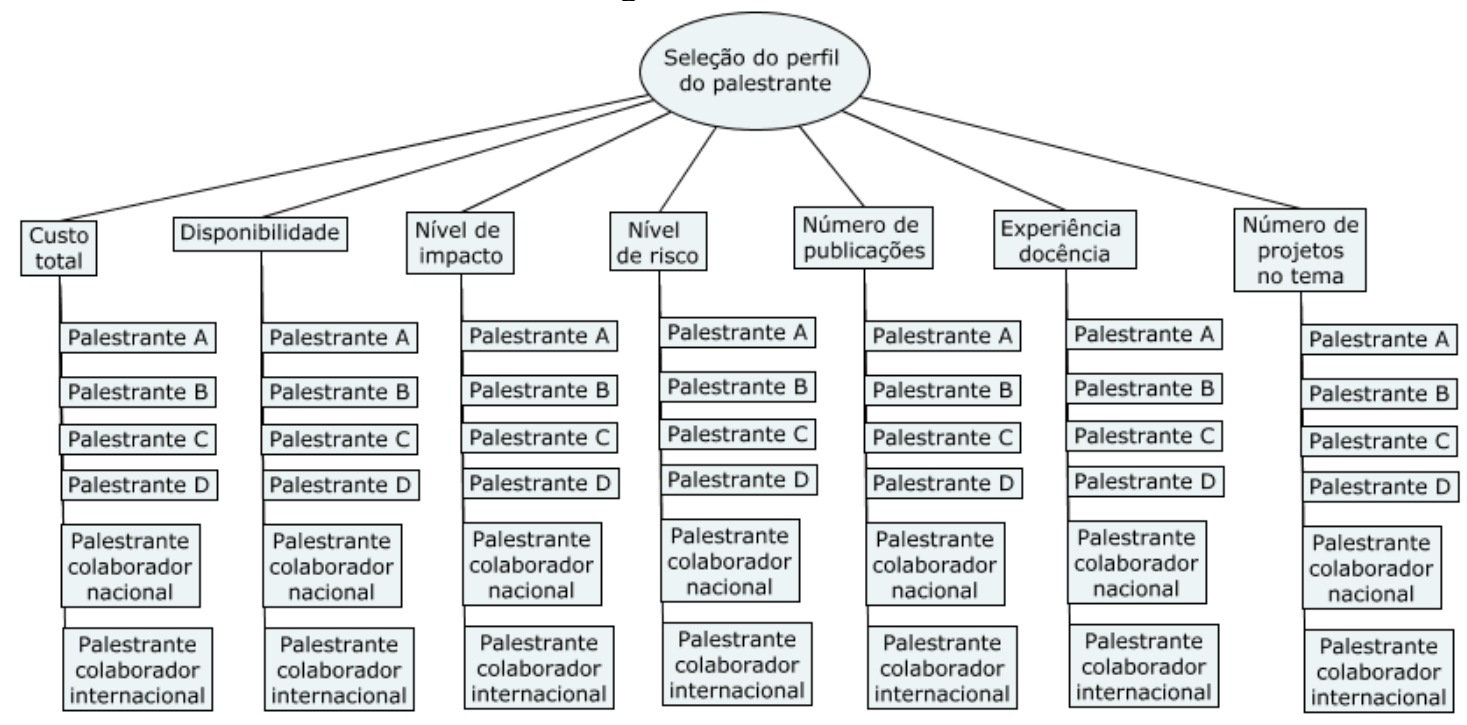

Fonte: Elaborado pelos autores.

Quanto aos critérios, com base no questionário e objetivos identificados foram definidos quais os mais relevantes e respectiva métrica de desempenho, conforme Tabela 3.

Tabela 3: Métrica de avaliação dos critérios.

\begin{tabular}{|c|c|}
\hline Critério & Métrica \\
\hline Menor custo Total & $\mathrm{R} \$$ \\
\hline Maior disponibilidade & 0 a 5 dias \\
\hline Maior nível de impacto & 0 a 10 \\
\hline Menor nível de risco & 0 a 3 \\
\hline Maior número de publicações & 0 a 37 publicações \\
\hline Maior experiência em docência & 0 a 25 anos \\
\hline Maior número de projetos no tema & 0 a 10 projetos \\
\hline
\end{tabular}

Fonte: Elaborado pelos autores.

A seguir, as informações das alternativas para cada critério foram pesquisadas e organizadas na Matriz de Consequências, conforme Tabela 4. O software utilizado foi o FITradeoff, disponível na versão Windows vista, no link: http://fitradeoff.org/download/. Nesta fase, a análise foi concluída por meio de entrevista com o Coordenador do Projeto, principal interessado e demandante do serviço, para ordenamento de preferências.

Seguindo os passos do FITradeoff, foi realizada uma pesquisa inicial (currículos, valor de voos) e teve-se o apoio do Decisor principal para pontuação nos demais critérios (alguns valores são fictícios apontados para distinção de um candidato a outro). Há também uma distinção entre a busca de valores mínimos (Custo Total e Risco) e valores máximos (Disponibilidade, Impacto, Número de publicações no tema, Experiência docência e Número de projetos no tema). 
Tabela 4: Matriz de Consequência do problema.

\begin{tabular}{|c|c|c|c|c|c|c|c|}
\hline $\begin{array}{c}\text { Alternativa/ } \\
\text { Critério }\end{array}$ & $\begin{array}{c}\text { Custo } \\
\text { total }\end{array}$ & Disponibilidade & Impacto & Risco & $\begin{array}{c}\text { Número de } \\
\text { Publicações } \\
\text { no tema }\end{array}$ & $\begin{array}{c}\text { Experiência } \\
\text { docência }\end{array}$ & $\begin{array}{c}\text { Número } \\
\text { de } \\
\text { projetos } \\
\text { no tema }\end{array}$ \\
\hline $\begin{array}{c}\text { Palestrante } \\
\text { A - nacional }\end{array}$ & 1390 & 2 & 6 & 1 & 15 & 10 & 4 \\
\hline $\begin{array}{c}\text { Palestrante } \\
\text { B - nacional }\end{array}$ & 2250 & 3 & 7 & 1 & 18 & 6 & 3 \\
\hline $\begin{array}{c}\text { Palestrante } \\
\text { C - } \\
\text { internacional }\end{array}$ & 34550 & 5 & 8 & 2 & 37 & 25 & 10 \\
\hline $\begin{array}{c}\text { Palestrante } \\
\text { D - } \\
\text { internacional }\end{array}$ & 23820 & 4 & 9 & 1 & 32 & 15 & 7 \\
\hline $\begin{array}{c}\text { Palestrante } \\
\text { convidado - } \\
\text { nacional }\end{array}$ & 100 & 1 & 5 & 3 & 15 & 5 & 1 \\
\hline $\begin{array}{c}\text { Palestrante } \\
\text { convidado - } \\
\text { internacional }\end{array}$ & 200 & 2 & 6 & 3 & 20 & 5 & 2 \\
\hline
\end{tabular}

Fonte: Elaborado pelos autores.

O Decisor (Coordenador do Projeto) definiu a ordem de preferência dos critérios (Figura 5). A ordem foi: Impacto $>$ Risco $>$ Custo Total $>$ Disponibilidade $>$ Número de projetos no tema > Número de publicações no tema $>$ Experiência docência.

Figura 5: Ordem de preferência dos critérios.

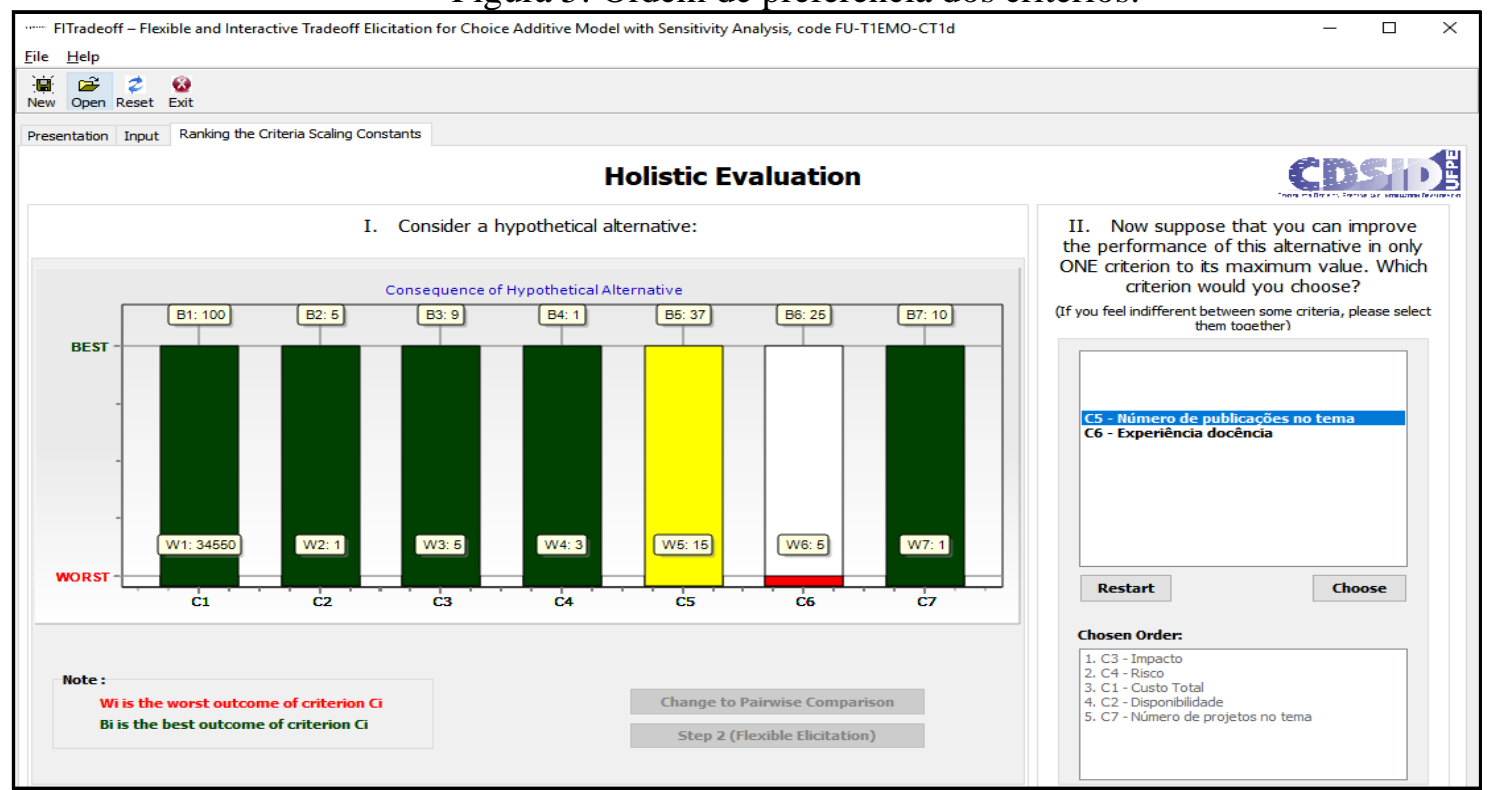

Fonte: Resultado do software FITradeoff.

O método FITradeoff apresenta ao decisor duas consequências para que se defina uma relação de preferência. Esta etapa do método é denominada Elicitação Flexível das Preferencias, a fim de minimizar as questões perguntadas para o Decisor, conforme Figura 6.

Este é considerado um método flexível, a ponto de o Decisor declarar se deseja continuar ou não o processo de elicitação (caso o resultado parcial já atenda ou ele não seja apto para decidir, por exemplo), tendo as opções de declarar a preferência dentre as alternativas, indiferença entre estas, ou simplesmente pular a questão. 
Figura 6: Escolha de consequências.

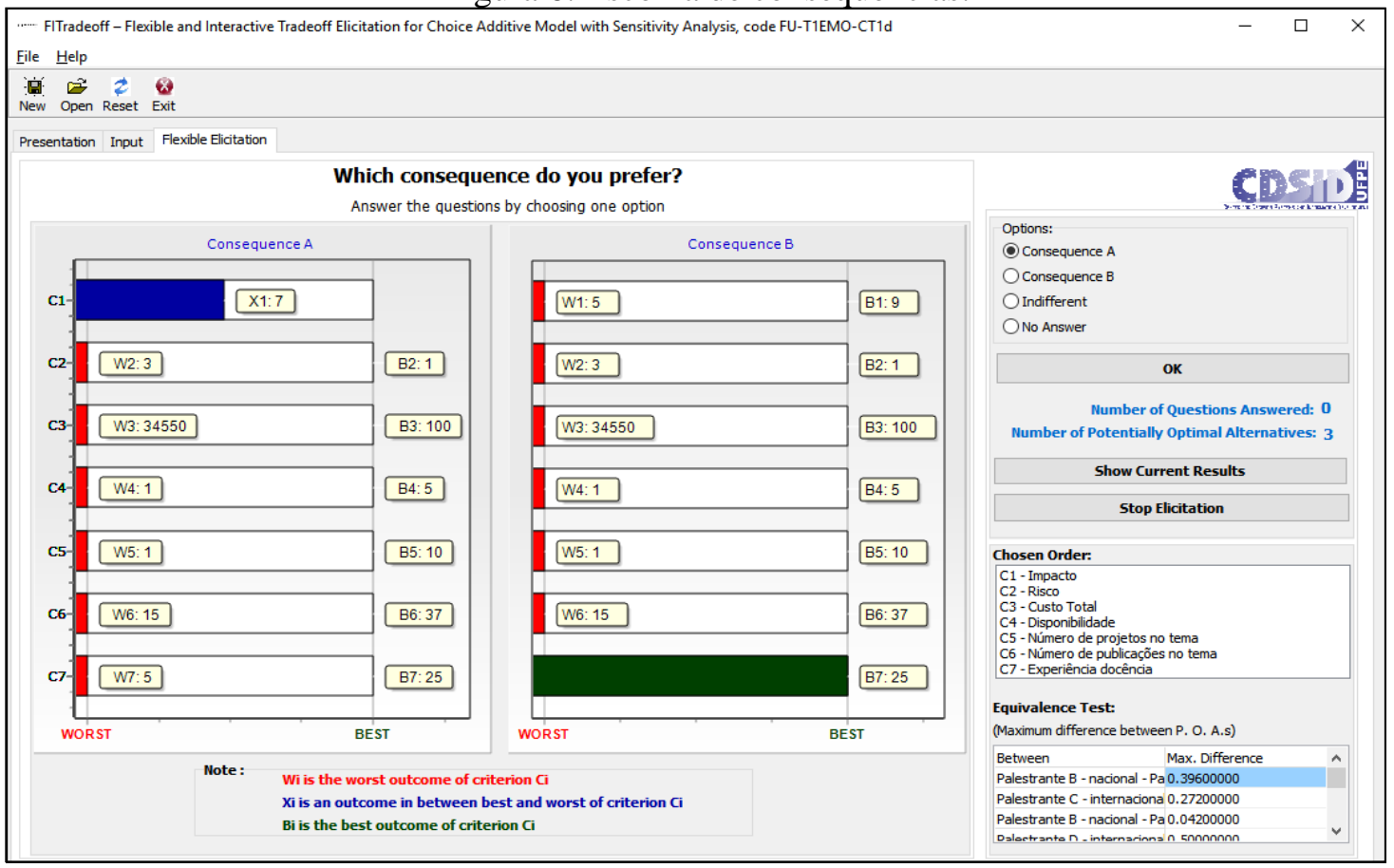

Fonte: Resultado do software FITradeoff.

Neste estágio são apresentadas várias questões de preferência, por exemplo, entre Impacto (primeiro critério) e Experiência docência (último critério) qual se daria mais relevância, segundo o Decisor seria a consequência A, Impacto. Os resultados são apresentados no Tabela 5.

Tabela 5: Ordem de escolha no software FITradeoff.

\begin{tabular}{|c|l|l|}
\hline Ciclo & "Qual consequência você prefere?" & Escolha \\
\hline 1 & Impacto x Experiência docência & Impacto (consequência A) \\
\hline 2 & Impacto x Risco & Impacto (consequência A) \\
\hline
\end{tabular}

Fonte: Elaborado pelos autores.

No total foram 2 questões, em uma comparação de critérios, por meio da principal pergunta: "Qual consequência você prefere?". Ao responder a primeira pergunta, do processo de elicitação, informando que a consequência $A$ era preferível a consequência $B$, o sistema foi capaz de encontrar a melhor alternativa. Diante desta aplicação foi possível constatar a flexibilidade e interatividade do método FITradeoff, considerando que o método e o sistema permitiram exigir pouco esforço cognitivo do decisor.

Ao final, tem-se o desempenho das alternativas em gráficos (Figura 7), sendo possível observar um gráfico com os valores máximo e mínimo dos critérios, destacando sobretudo o critério de "Impacto". 
Figura 7: Gráfico do processo de escolha de preferência.

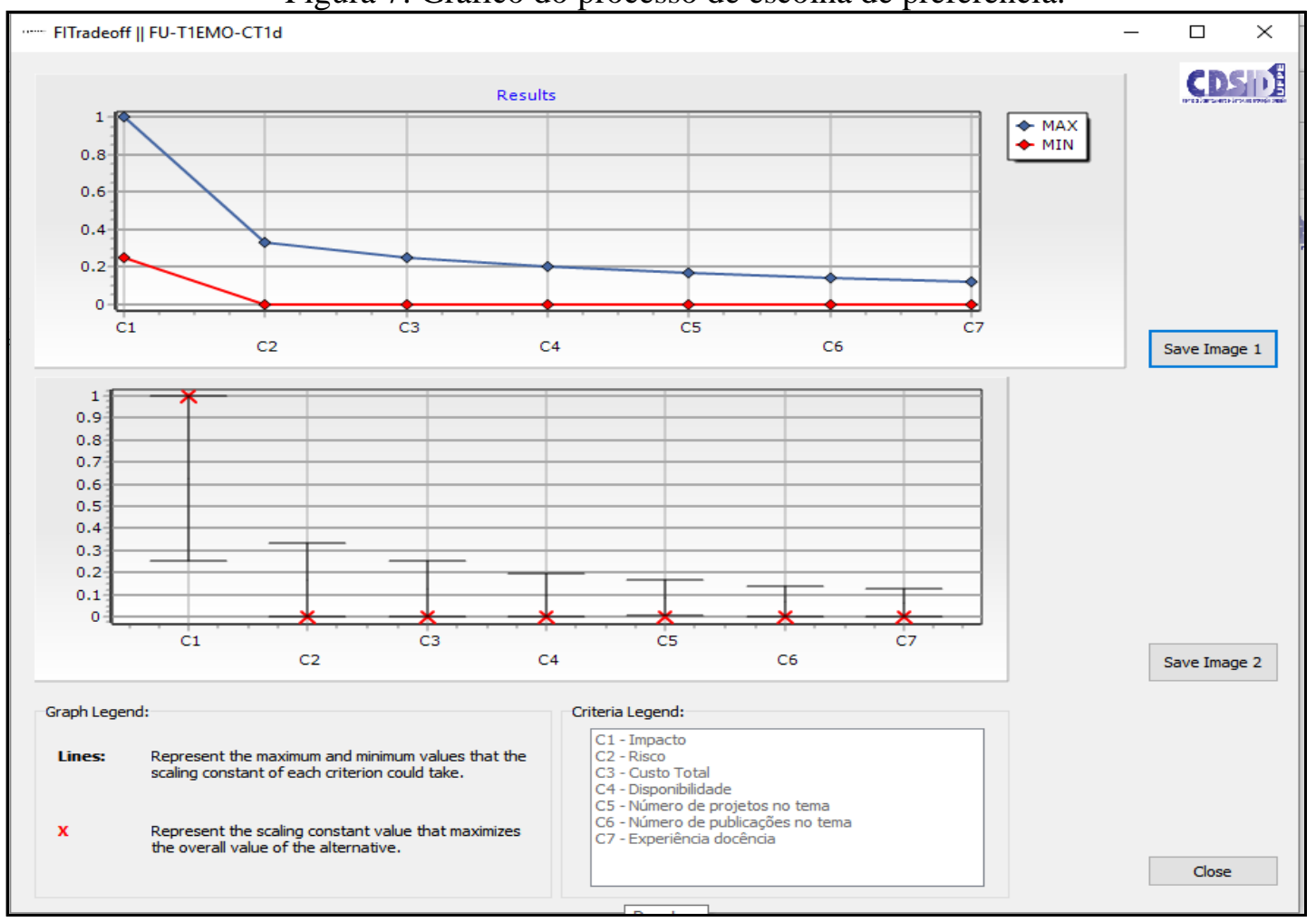

Fonte: Resultado do software FITradeoff.

A solução para o problema segundo o modelo de preferências foi a alternativa "Palestrante D - internacional", que melhor atenderia a demanda (Figura 8). Este perfil é o do Professor do MIT, com disponibilidade de 4 (quatro) dias, impacto 9 (considerado alto na escala de 0 a 10), risco baixo, custo total estimado (passagem, valor hora, hospedagem, alimentação e transporte) de $\mathrm{R} \$ 23.820,00$.

Figura 8: Melhor alternativa.

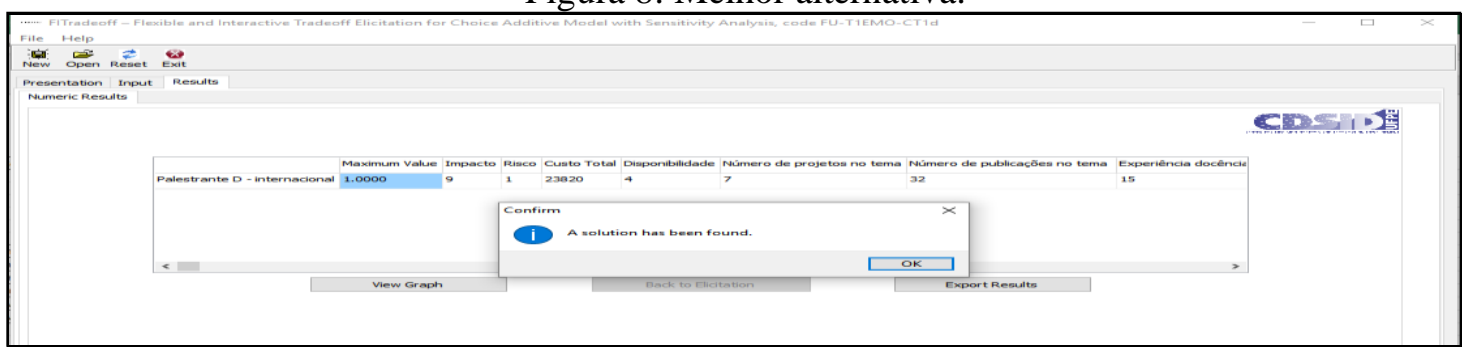

Fonte: Resultado do software FITradeoff.

Em suma, é possível analisar no FITradeoff que, após a definição da ordem de preferência de todos os critérios, foi questionado a preferência entre "Impacto" e "Experiência docência" (primeiro e último critério da listagem anterior), sendo os melhores "Palestrante C nacional" e "Palestrante D - internacional". Quando "Impacto" é selecionado pelo decisor, ganha o "Palestrante D - internacional".

A segunda questão compara "Impacto" e "Risco" (destacando-se "Palestrante A nacional", "Palestrante B - nacional" e novamente "Palestrante D - internacional"), sendo escolhido o critério "Impacto", o que resulta no mesmo ganhador.

Observa-se também que na comparação de critérios (exceto "Custo total" e "Risco"), na Tabela 4, o "Palestrante C - internacional" e o "Palestrante D - internacional" apresentam valores próximos e nas melhores colocações. Destes dois, o "Palestrante D - internacional" apresenta o menor "Custo total" e "Risco". 
Assim, é possível observar que as questões consideram a ordem de preferência dos critérios, caso o decisor divirja e escolha uma ordem diferente entre critérios é realizado nova rodada de questões até a melhor alternativa. Essa análise pode servir para estudo de diferentes tipos de cenários, comparando os critérios mais relevantes para um ou mais decisores.

Observa-se também que um grupo diferente de decisores (ou mesmo mais decisores do que os envolvidos neste trabalho) poderia resultar em outro perfil de palestrante, pois o método FITradeoff segue a estrita escolha do decisor.

\section{Conclusões}

Métodos flexíveis e interativos para apoio a tomada de decisão são procurados para resolução de problemas desde simples a complexos, de modo a abranger multicritérios e diferentes alternativas.

A contribuição desta pesquisa está no estudo de uma estrutura e respectivos métodos para análise do problema, definição de critérios e alternativas, até o apoio na escolha da melhor alternativa ótima.

Ao considerar a necessidade de critérios bem definidos em processos de seleção pública ou privada foram estudados os métodos MCDA, para uma visão geral e estruturação do problema em estudo, e definidos os métodos: 1) VFB (Value Focused Brainstorming) para a estruturação do problema, bem como identificar e alinhar objetivos, critérios e alternativas. 2) FITradeoff (Flexible and Interactive Tradeoff) que permite a comparação de alternativas para melhorar o processo de licitação e exigir menos informações do decisor. Assim, cada método contribuí em uma parte da estrutura da análise.

Destaca-se que a escolha dos métodos foi determinada com base no foco da pesquisa e problemática, sendo a seleção do perfil do palestrante especialista, busca-se identificar mais alternativas e dentre estas selecionar a ótima.

Como ponto fraco, cada método contribui parcialmente na análise, mas considerando que cada problema exige um raciocínio focado, cada método tem sua característica própria para atender, sendo necessário adaptações ou até integração de métodos.

Ressalta-se que há a necessidade de praticidade no processo decisório, e com o VFB foi possível encontrar os objetivos, delinear os critérios e as alternativas; esta foi considerada a tarefa mais trabalhosa na análise. Já com o FITradeoff, considerado um método novo, foi possível analisar as alternativas de maneira mais fácil e mantendo a eficácia. Por fim, a integração destes métodos se mostrou eficiente e eficaz, com resultados destacados neste artigo.

Este estudo teve por base o contexto de contratação de profissional para promover uma oficina docente em uma Universidade, por processo licitatório, para abordar o tema CDIO para aperfeiçoamento de técnicas docentes. Ao final, o objetivo desta análise foi atingido e os resultados foram apresentados aos Stakeholders, que validaram e responderam como satisfatório, além de apontar como benefícios o processo de estabelecimento de objetivos, definição de critérios e a maior facilidade no processo de seleção da alternativa ótima.

Além disso, é válido destacar que na maioria das situações vividas e relatadas pelos decisores, não há muito tempo hábil para dedicação na montagem de um plano estratégico, com pensamento estruturado nos critérios de seleção e estudo profundo da real necessidade. Assim, estudos voltados a análise de decisão, como realizado neste artigo, são considerados válidos, pois conduzem à pesquisa de como realizar a estruturar e solucionar a situação problemática, trazendo mais confiança ao decisor, evitando desperdício de recursos (tempo, pessoal) e retrabalhos por decisões equívocas e consequências indesejadas.

Quanto a limitações, há o nível de conhecimento dos Stakeholders sobre o assunto e o tempo disponibilizado.

Para futuros trabalhos sugere-se: 1) Outras aplicações com os métodos VFB e FITradeoff, para base de decisões com recursos públicos e privados, visando facilitar o processo para decisão mais consciente e gestão eficaz; 2) Combinações ou comparações com outros métodos da área de MCDA, PSM ou gestão de projetos poderiam ser estudadas. 
Agradecimentos. Os autores agradecem o apoio da Coordenação de Aperfeiçoamento de Pessoal de Nível Superior (CAPES).

\section{Referências}

Abuabara, L., Paucar-Caceres, A., Neyra Belderrain, M. C. e Burrowes-Cromwell, T. A systemic framework based on Soft OR approaches to support teamwork strategy: An aviation manufacturer Brazilian company case. Journal of the Operational Research Society, v. 69, n. 2, p. 220-234, 2018.

Almeida, A. T. Processo de Decisão nas Organizações: Construindo Modelos de Decisão Multicritério. São Paulo: Atlas, 2013.

Almeida, A. T., Cavalcante, C. A. V., Alencar, M. H., Ferreira, R. J. P., Almeida-Filho, A. T. e Garcez, T. V. Multicriteria and Multiobjective Models for Risk, Reliability and Maintenance Decision Analysis. Nova York: Springer International Publishing, 2015.

Almeida, A. T., Almeida, J. A., Costa, A. P. C. S. e Almeida-Filho, A. T. A new method for elicitation of criteria weights in additive models: Flexible and interactive tradeoff. European Journal of Operational Research, v. 250, n. 1, p. 179-191, 2016a.

Almeida, J. A., Frej, E. A. e Almeida, A. T. Heurísticas aplicada ao método FITradeoff para reduzir o número de perguntas do procedimento de elicitação de pesos. In: Anais do XLVIII Simpósio Brasileiro de Pesquisa Operacional, Vitória. SOBRAPO, 2016b. p. 4083-4086.

Andrade, A. L. O. e Morais, D. C. O uso do método de estruturação de problema Strategic Choice Approach no desenvolvimento de novos produtos. In: Anais do XXXVI Encontro Nacional de Engenharia de Produção, João Pessoa. ABEPRO, 2016. p. 1-13.

Bana e Costa, C. A. e Vincke, P. Multiple criteria decision aid: An overview. In: Bana e Costa, C. A. (ed.). Readings in Multiple Criteria Decision Aid, cap. 1, p. 3-14. Berlim: Springer Berlin Heidelberg, 1990.

Camilo, D. G. G., Souza, R. P., Frazão, T. D. C. e Costa Junior, J. F. Multi-criteria analysis in the health area: selection of the most appropriate triage system for the emergency care units in natal. BMC Medical Informatics and Decision Making, v. 20, n. 1, p. 38-53, 2020.

Figueira, J., Greco, S. e Ehrgott, M. (eds.) Multiple Criteria Decision Analysis: State of the Art Surveys, $2^{\mathrm{a}}$ ed. Nova York: Springer, 2016.

Franco, L. A. e Montibeller, G. Problem structuring for multicriteria decision analysis interventions. In: Wiley Encyclopedia of Operations Research and Management Science, cap. 1, p. 7. Hoboken: John Wiley \& Sons, 2011.

Frej, E. A. Método multicritério de elicitação por tradeoff interativo e flexível para a problemática de ordenação e para a tomada de decisão em grupo.100 f. Tese (Doutorado em Engenharia de Produção) - Centro de Tecnologia e Geociências, Universidade Federal de Pernambuco, Recife-PE, 2019.

Frej, E. A., Roselli, L. R. P., Araújo de Almeida, J. e Almeida, A. T. A multicriteria decision model for supplier selection in a food industry based on FITradeoff method. Mathematical Problems in Engineering, v. 2017, ID 4541914, 2017.

Gonçalves, T. J. M. Adaptação do value focused thinking à identificação de objetivos de 
desempenho da qualidade. In: Anais do XVII Simpósio de Engenharia de Produção, Bauru. UNESP, 2010. p. 1-16.

Holanda, N. E. C. Modelo para seleção de uma ferramenta de TI com foco em produtividade: uma aplicação baseada no VFT e FITradeoff. $128 \mathrm{f}$. Dissertação (Mestrado em Engenharia de Produção) - Centro Acadêmico do Agreste, Universidade Federal de Pernambuco, Caruaru-PE, 2017.

Keeney, R. L. Value Focused Thinking: A path to creative decision making. Cambridge: Harvard University Press, 1992.

Keeney, R. L. Creativity in decision making with value-focused thinking. Sloan Management Review, v. 35, n. 4, p. 33-41, 1994.

Keeney, R. L. Value-focused thinking: Identifying decision opportunities and creating alternatives. European Journal of Operational Research, v. 92, n. 3, p. 537-549, 1996.

Keeney, R. L. Value-focused brainstorming. Decision Analysis, v. 9, n. 4, p. 303-313, 2012.

Keeney, R. L. e Raiffa, H. Decisions with Multiple Objectives: Preferences and value tradeoffs. Cambridge: Cambridge University Press, 1993.

Macedo, P. P., Mota, C. M. M. e Sola, A. V. H. Meeting the Brazilian energy efficiency law: A flexible and interactive multicriteria proposal to replace non-efficient motors. Sustainable Cities and Society, v. 41, p. 822-832, 2018.

Madeira, A. E. e Palmeira, E. M. Indicadores de desempenho na gestão de contratos de serviços. Contribuciones a La Economía, v. 19, 2011.

Mingers, J. e Rosenhead, J. Problem structuring methods in action. European Journal of Operational Research, v. 152, n. 3, p. 530-554, 2004.

Poleto, T., Clemente, T. R. N., Gusmão, A. P. H., Silva, M. M. e Costa, A. P. C. S. Integrating value-focused thinking and FITradeoff to support information technology outsourcing decisions. Management Decision, v. 58, n. 11, p. 2279-2304, 2020.

Silva, M. M., Gusmão, A. P. H., Andrade, C. T. A. e Silva, W. The integration of VFT and FITradeoff multicriteria method for the selection of WCM projects. In: Anais da 2019 IEEE International Conference on Systems, Man and Cybernetics (SMC), Bari, Itália. IEEE, 2019. p. 1513-1517.

Souza Junior, V. P., Belderrain, M. C. N., Braguini, C. E. M., Carvalho, N. H. C. e Araújo, P. F. Escolha de um município para implantação de um centro de formação profissional utilizando um método de apoio multicritério à decisão. Revista Gestão em Engenharia, v. 2, n. 1, p.13-26, 2015. 